Copyright (C) 2021 by Cherkas Global University

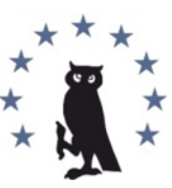

Published in the USA

European Journal of Physical Education and Sport

Has been issued since 2013 .

E-ISSN: 2409-1952

2021. 9(1): 32-41

DOI: 10.13187/ejpe.2021.1.32

https://ejpes.cherkasgu.press

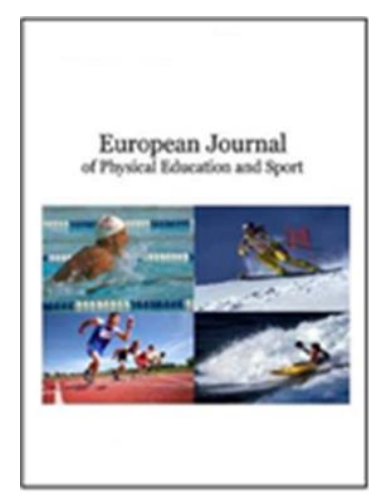

\title{
The Bridge between Abstract Biomechanics and Tennis Strokes
}

\author{
Konstantinos G. Papageorgiou a , * \\ a Ionian University, Greece
}

\begin{abstract}
Abstract biomechanics, by definition, are biomechanics in theory, without any application (therefore a geometrical representation of movement). When referring to (abstract) biomechanics and to (applied) technique ("technical form") their relationship is blurred, not to mention there is no method to effectively make the transition from the former to the latter. In this paper, these terms are explicated and the connection between them is restored through a bridge, called the operating principle or mechanism of the technique. The exemplary field of application is the sport of tennis. In tennis, the operating principle is not of course all that is needed to develop technical form (the application of the theoretical model on one's body). It is the first step from at least three more, the other ones being the "reference points" and the shot teaching mechanism (the latter being substantially different from the mechanism of the technical form presented here). However, the idea of the operating principle (of the technical form) has never been presented before for any sport. The implications of these insights are important both methodologically and for the development of a teaching method for tennis technical form. Finally, this paper itself bridges two gaps: theory to practice and epistemology to science.
\end{abstract}

Keywords: technique, operating principle, biogeometrical model, methodology, structure.

\section{Introduction}

Abstract biomechanics is the application of mathematical modelling to the mechanics of biological effectors resulting in archetypical, or mathematical, descriptions of movement (abstract structure $^{*}-c f$. Gr. domē). When such descriptions are expressed as actual movements by e.g. humans (in the case of sports), these resulting movement are called the technical form, or simply, the technique (concrete structure - $c f$. Gr. domèma). But how do we get from the abstract model to the actual (concrete) tennis shot? We go through bridging principles (abstract layout of the process of structuring - cf. Gr. domēke $)$. These principles are what this paper will cover. The way this transition from abstract structure to concrete structure is practiced is called training (the actual process of structuring/building - cf. Gr. domēsē). In this process of domēse one may also find the idea of reference points which function as a kind of physical anchors for the easier and more precise acquisition of the technique (discussed in more detail in: Papageorgiou, 2020c). Finally, one also needs the "essence" of the teaching method, called the shot-teaching mechanism, which is a key-constituent of the learning process. The general interconnection of the various parts of the

\footnotetext{
${ }^{*}$ Corresponding author

E-mail addresses: cconstantinoss@gmail.com (K.G. Papageorgiou)

* As is explained in this paragraph, for the four distinct concepts related to Lat. structura, Greek language has four words instead of one. Latin-derived languages miss this symmetry and this is oftentimes expressed as deficiency in their theoretical structures.
} 
teaching method partly discussed here are presented in (Papageorgiou, 2020e). Training in order to become a motor expert is the content of the Distal Method and has been covered in various papers (Papageorgiou, Papadopoulos, 2018; Papageorgiou, 2019, 2020a, 2020d).

This paper is based on the specific methodological conception about sports biomechanics presented in an earlier article (Papageorgiou, 2020b). According to this Classical Epistemological approach, we do not make biomechanical models for technique by means of observation or by copying professional players. What we should do is select certain, initial conditions (by whatever criterion we deem appropriate) and develop a model irrespective of the "real world". After we finish developing it in abstraction, we may go back to the world and try to fit our observations to our model - without altering the model, since it is forbidden to verify or to falsify theoretical models by means of observation. This might not be in line with some popular, save delusional conception of science, but it is in line with how mathematics work, implying here that some popular, save superficial interpretation of science and mathematics are incompatible as far as methodology is concerned. Logic, which is the very foundation of both mathematics and science, forbids us to go from the effect (observation) to the cause (theory), a logical fallacy known as "begging the question". Another obstacle confusing us about the relationship between theories and the world is what we mean in English when referring to "structure": ideal structure or concrete structure; dome, or domèma?

When it comes to tennis biomechanics, the main article presenting the biomechanical model for tennis was Papageorgiou, 2016. Taking into consideration the basic biomechanical principles, it analysed the contact point (or contact "period") in a serial kinetic chain. The main consideration was how to maximise the kinetic energy transfer to the ball during that period. This was called Critical Force Maximisation (CFM). Some important conclusions about CFM were:

- Using the wrist was discouraged

- Last joints were actively decelerated to induce the whip effect

- A kind of double kinetic chain was encouraged. In this case the serial kinetic chain starting from the hips was coordinated with the forward weight transfer.

Building on that, we shall see how the elemental biomechanical components of basic tennis strokes play out. The purpose is to bridge the gap between biomechanics and technical form; however, we shall see neither biomechanics nor technical form but only the bridging principle that connects them. It is the first time such a bridging principle is proposed.

This bridging principle is also called the operating principle (or "mechanism") of each tennis shot. As the operating principle of shots, we define the main and most efficient mechanism of producing kinetic energy that will be used to achieve the CFM upon contact. The mechanism is fundamental, simple and universal, i.e. may be applied to different technical models. Next, we shall see the mechanisms for forehand \& backhand (one and two-handed) drives, service (a basic flat serve) and underspins. The applied model presented here is biogeometrical, i.e. some interpretation of geometry on human movement. Without getting into too much detail in this paper, we shall also see some examples of how the operating principles are transformed to tennis shots.

\section{Results and discussion}

\section{From biomechanics to tennis shots}

In the beginning of biomechanics, there are the mathematical models. The models themselves do not dictate their application; mathematical models are fully abstract with no meaning whatsoever for the reality (this is why a certain model may be used now in physics and in a million years in economics as well). The said mathematical models, the ones used by biomechanics, have been developed in abstraction without any concern for the world or for their future applications or their non-applications. This is the case for any other mathematical model as well. Only afterwards do we assign phenomena to mathematical models. This way, there will always be errors; however, the errors are not mathematical in their nature (the abstract model is never wrong) but practical (bad fit between the model and the phenomenon). So, we still use other mathematical models (e.g. from approximation theory or from statistics) to deal with the world's uncertainty (which is not a mathematical uncertainty).

A direct, or indirect, application of mathematical models, but also of physiology, is the production of a series of "biomechanical principles", such as the applied Newton's Laws, Range of Motion, the Kinetic Chain etc. (Bloomfield, Ackland, 1994; Knudson, 2007). These principles are in 
between the world and the full abstraction of theory. Therefore, such principles are ideal for us to bridge the gap between theory and practice, and to be used as the basis of our own bridging/operating principles.

The main two principles that are of interest to us here are, the Biomechanical Advantage, which refers to efficient body positions, and the Kinetic Chain principle stating that each movement is the result of a certain coordination pattern of at least two body segments. These body segments are connected just like the different rings of a chain and movement is transmitted through them. This coordination pattern may be said to be serial, one-unit or mixed (or simply, uncoordinated!).

The operating principle concerns the most important part of that chain, the one that is more characteristic to the movement, so to speak. In the Distal Method, this segment is central for the successful development of the technical form, i.e. the application of biomechanics to a tennis shot. The reasons are:

- It concerns the closest segment to ball contact.

- It may be used as a steppingstone for the player to concentrate on while learning.

- It represents the simplest action performed in the corresponding tennis shot.

Why is the choice made here so strong? Why not some other movement? Because it is the simplest possible movement with the maximum biomechanical advantage and one does not need to "prove" why we begin from the simplest movement but, on the contrary, one should convincingly demonstrate, why there is a need to take, as a starting point, anything more complex than that.

For simplicity, the operating principle does not take into consideration the first body segments in a kinetic chain analysis and may equally work with unit or serial kinetic chains. Later on, while practicing the movement, the focus will also be on, how to coordinate the movement (described here as the operating principle) with the rest of the kinetic chain (and this is an implication for practice).

The presentation of the operating principles of three basic tennis shots will be attempted next. The mathematical models used in this paper are geometrical and their depiction is in the form of applications on the human body.

\section{Drives}

The arm is used as a catapult. There are two extreme cases, one moving in the (para)sagittal and one in the transverse (thoracic) plane (depending on the ball height). All other cases are combinations of these two extremes to various degrees. In the sagittal plane, the pivot point is on the frontal axis (at the height of the shoulder), whereas in the transverse plane the pivot point is the longitudinal axis. Figure 1 shows the simplest case of arm rotation in the sagittal plane.

In the simplest case (less Freedom Degrees - DOF) of the sagittal-plane movement, acceleration in the forward movement (that is of interest to us) is enhanced by:

- Body musculature (early phases)

- The gravity (medium phases)

- Whip effect (later stages)

The whip effect is the acceleration of the next, lighter segment of a kinetic chain when the previous segment rapidly decelerates. This will be more obvious in the technical interpretation of the biomechanical model. For now, the whip effect is simply induced by the very length of the arm. The arm is elastic and can both increase and decrease in length. This is under conscious control: we may touch a wall with our hand fully extended and then contract it without moving our body, and while keeping it fully extended, we do not touch the wall anymore (Figure 2). From then on, engaging previous body segments (e.g. legs) in proper synchronisation, will accelerate our arm even more.

In the transverse plane, all previous apply, except from the gravitational acceleration which now has no effect being a vertical force. However, the kinetic chain may provide all the necessary acceleration for equally effective strokes.

The cases of forehand and one-handed backhands are the same in that respect (Figure 3). Even two-handed backhands may be reduced to the aforementioned cases if we assume that the non-dominant arm executes the same movement as the dominant arm in all the one-handed cases. Note: for "winners" and other high shots the operating principle is similar but the arm moves on the horizontal plane (not shown here). 


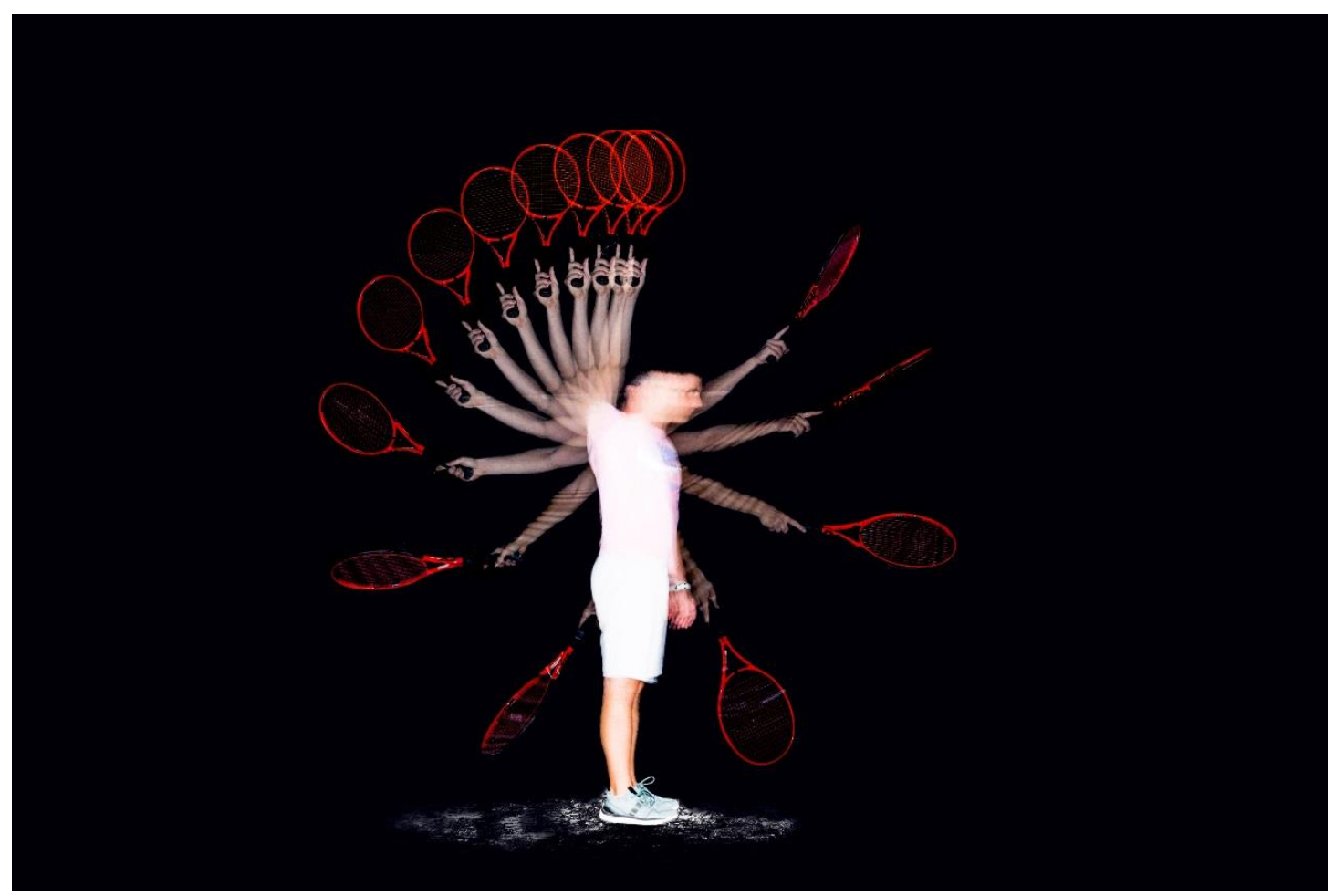

Fig. 1. The operating principle of forehand drive in the sagittal plane. The arm rotates counter clockwise

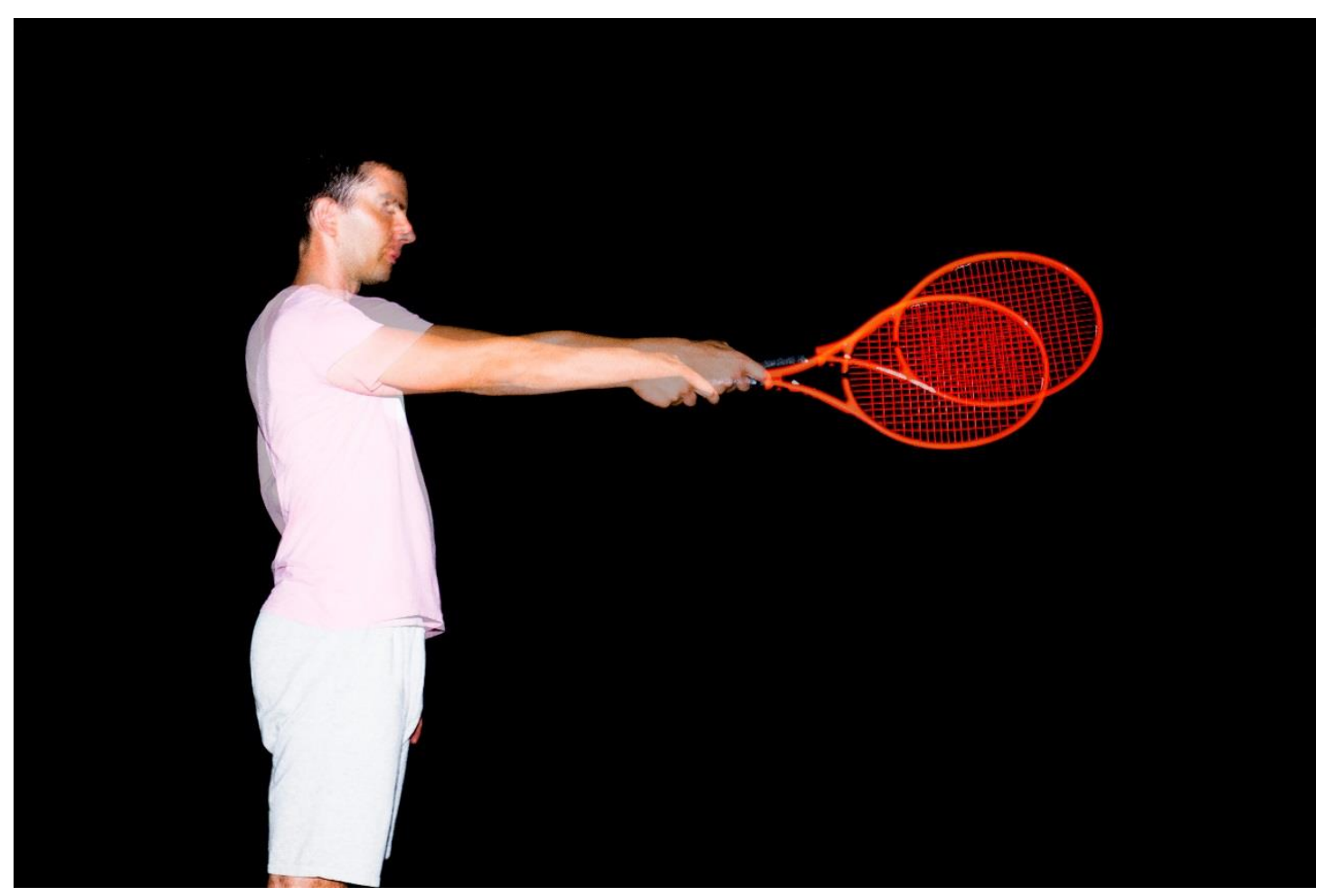

Fig. 2. The isolated reach of the arm 


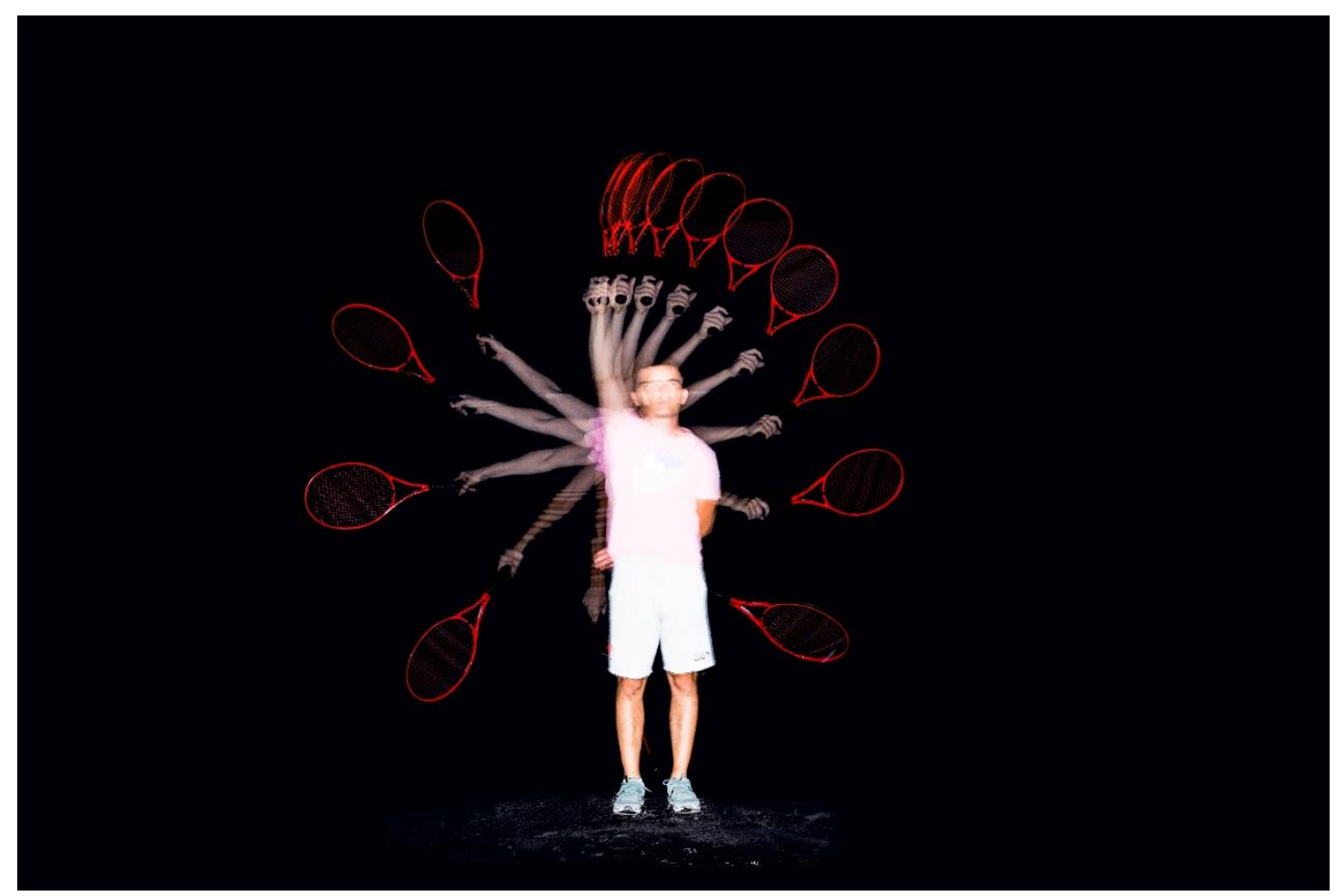

Fig. 3. The Operating Principle in one-handed backhand drive. The arm rotates clockwise in the frontal plane

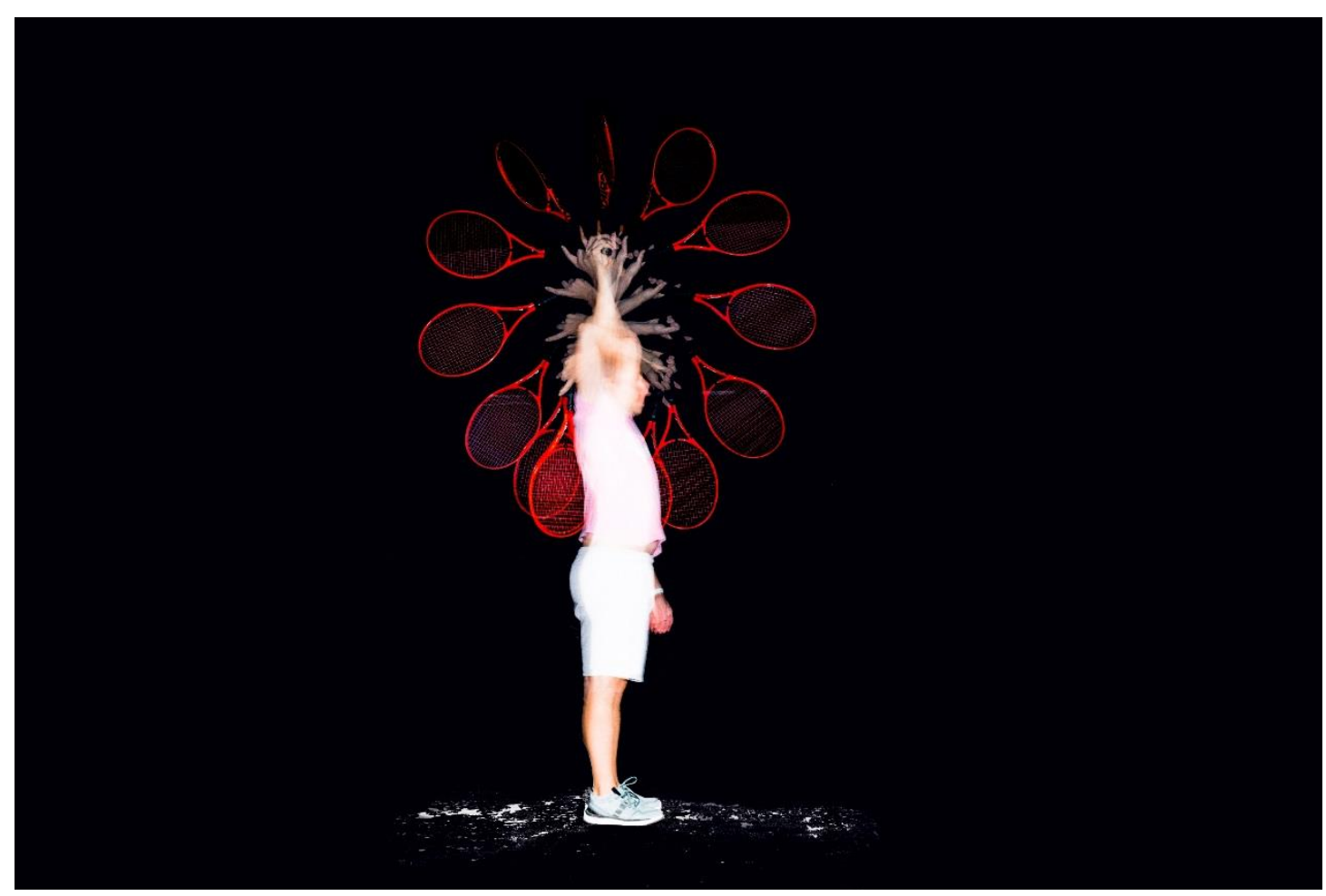

Fig. 4. $180^{\circ}$ pronation on the sagittal plane. The movement is clockwise and the elbow remains in the same height 


\section{Service}

We shall examine the case of a first, flat serve. In this case, the motion is on the sagittal plane. The frontal axis of rotation is on the level of the elbow; the elbow is located distally, in the maximum height of its trajectory due to the kinetic chain.

Because the body blocks the movement of the racket in the sagittal plane, the movement starts in the frontal (coronal) plane while the body is positioned sideways (in relation to the net). Halfway through the movement, the body changes direction to face the net. From then on, the movement of the lower arm-racket system unfolds in the sagittal plane.

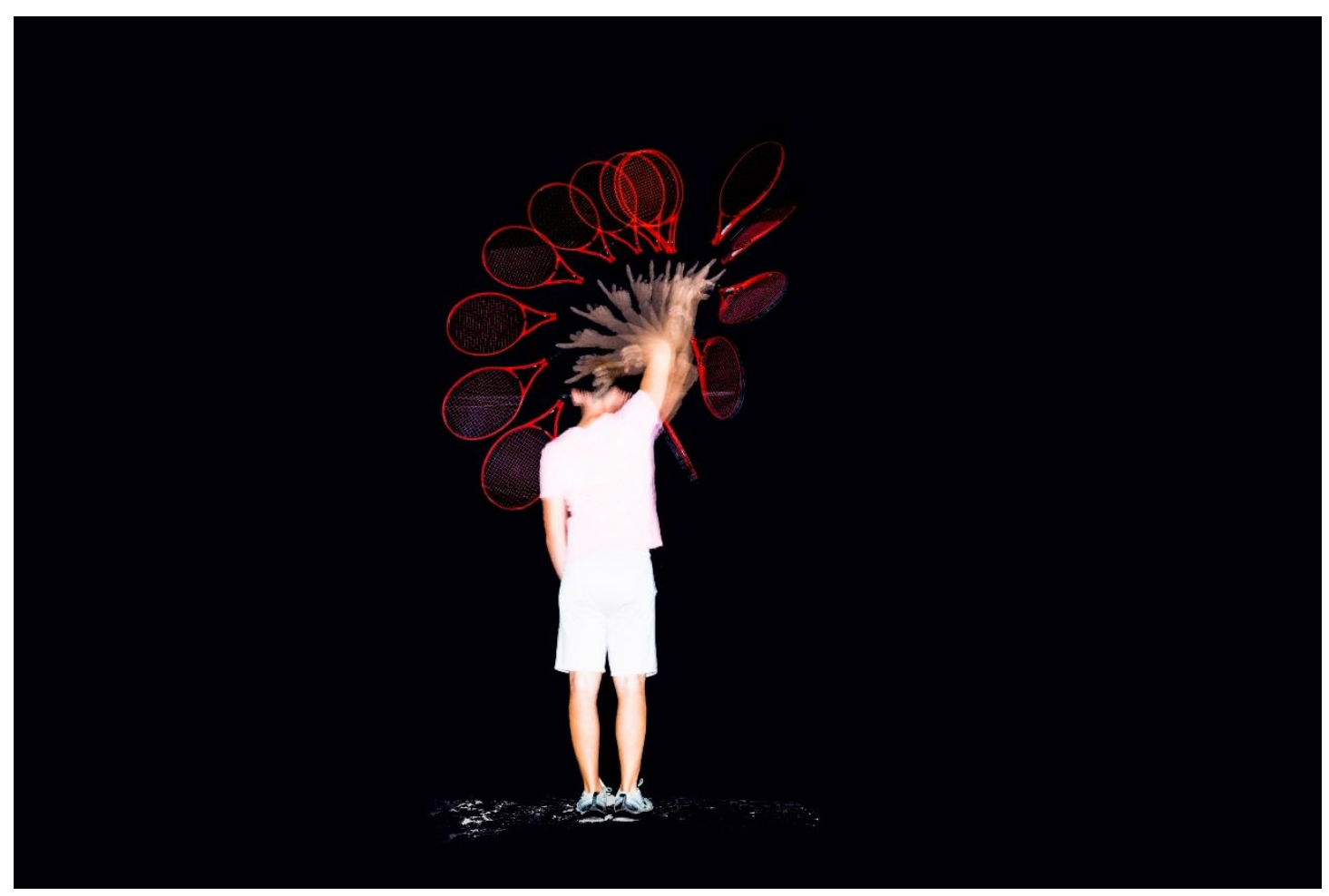

Fig. 5. Rotation (counter clockwise) in the frontal plane maintaining the elbow at the same height

\section{Underspins}

The underspins category includes various shots with the same kinesiology: volleys, drops slices. All of them have the same operating principle (their difference, apart from the different swing length, but not swing path, is solely on the inclination of the racket's face upon impact).

The operating principle of underspins is that of a pendulum. The arm is like the thread (straight) and the shoulder is the pivot point. The wrist is constantly fully extended and the face of the racket points upwards when the arm is in the vertical position, almost touching the body.

The movement of the arm is neither backward (sagittal plane) nor sideways (coronal plane). It is in between these two planes, so it is a diagonal movement going backwards and sideways. Figures 6 and 7 depict the operating principle for backhand underspin's and Figure 8 for forehand underspins. 


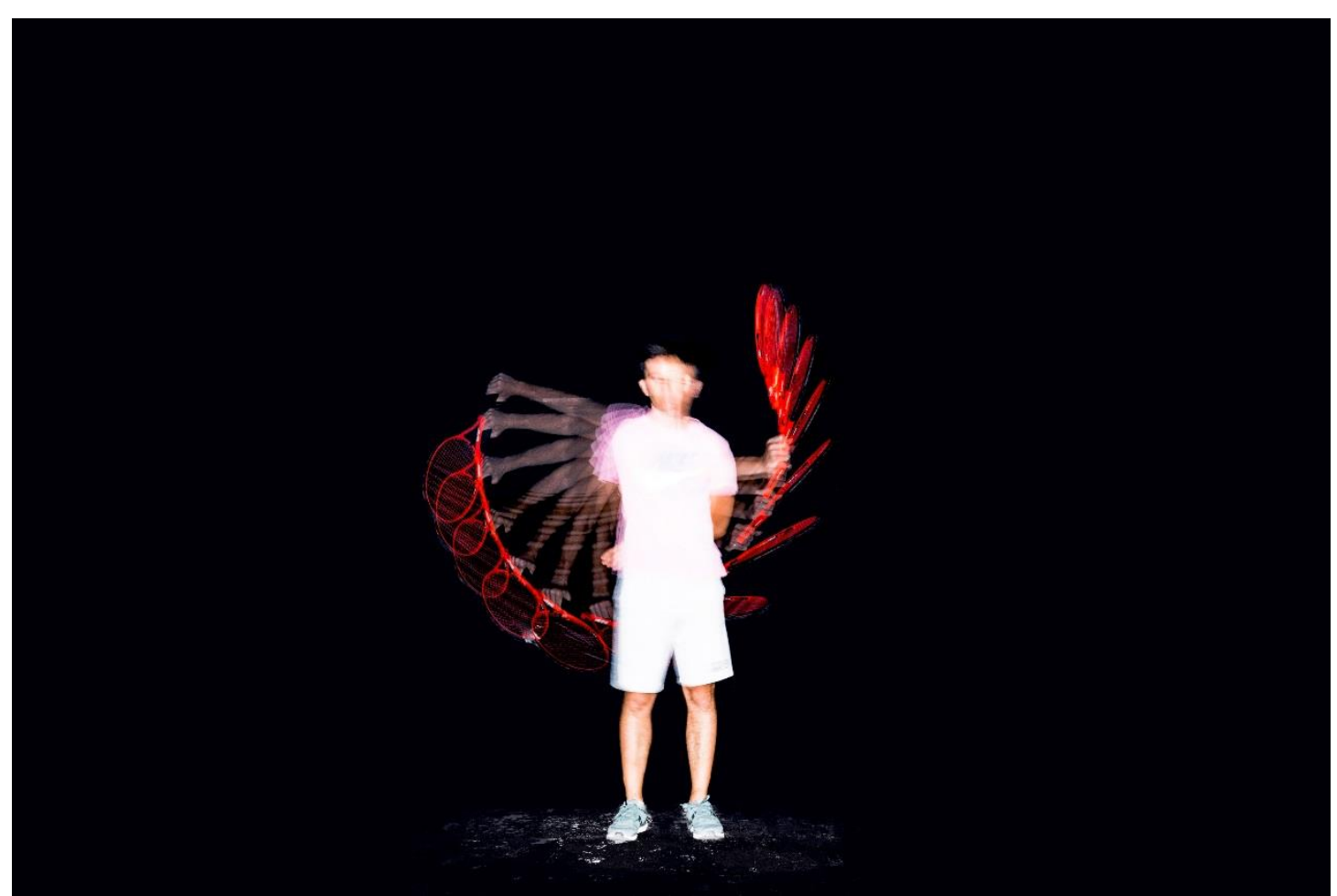

Fig. 6. The Operating Principle of backhand underspins. The rotation happens clockwise and the plane is an intermediate between the frontal and the sagittal plane

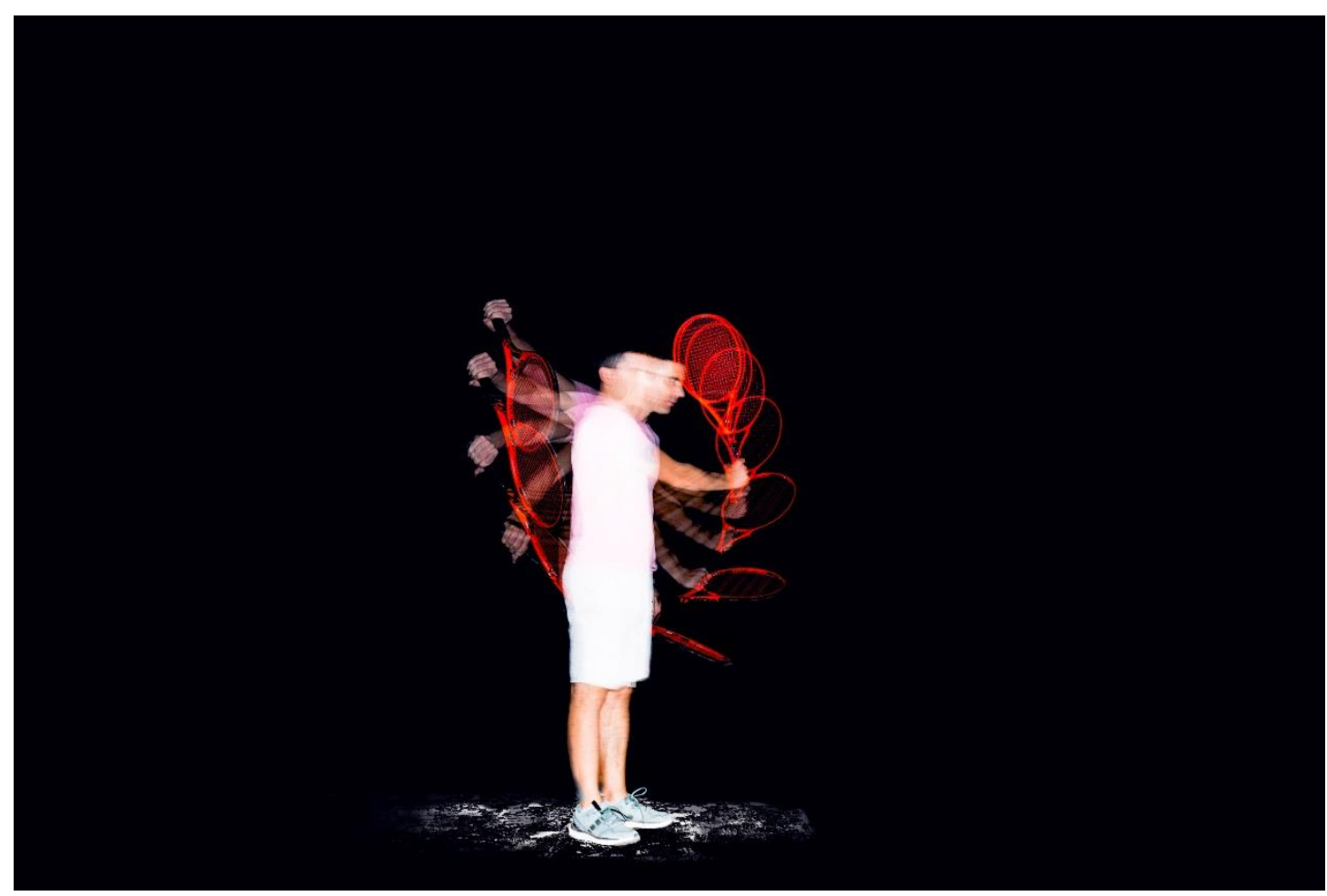

Fig. 7. The Operating Principle of backhand underspins seen from another angle where the rotation (clockwise) between the frontal and the sagittal plane is more evident 


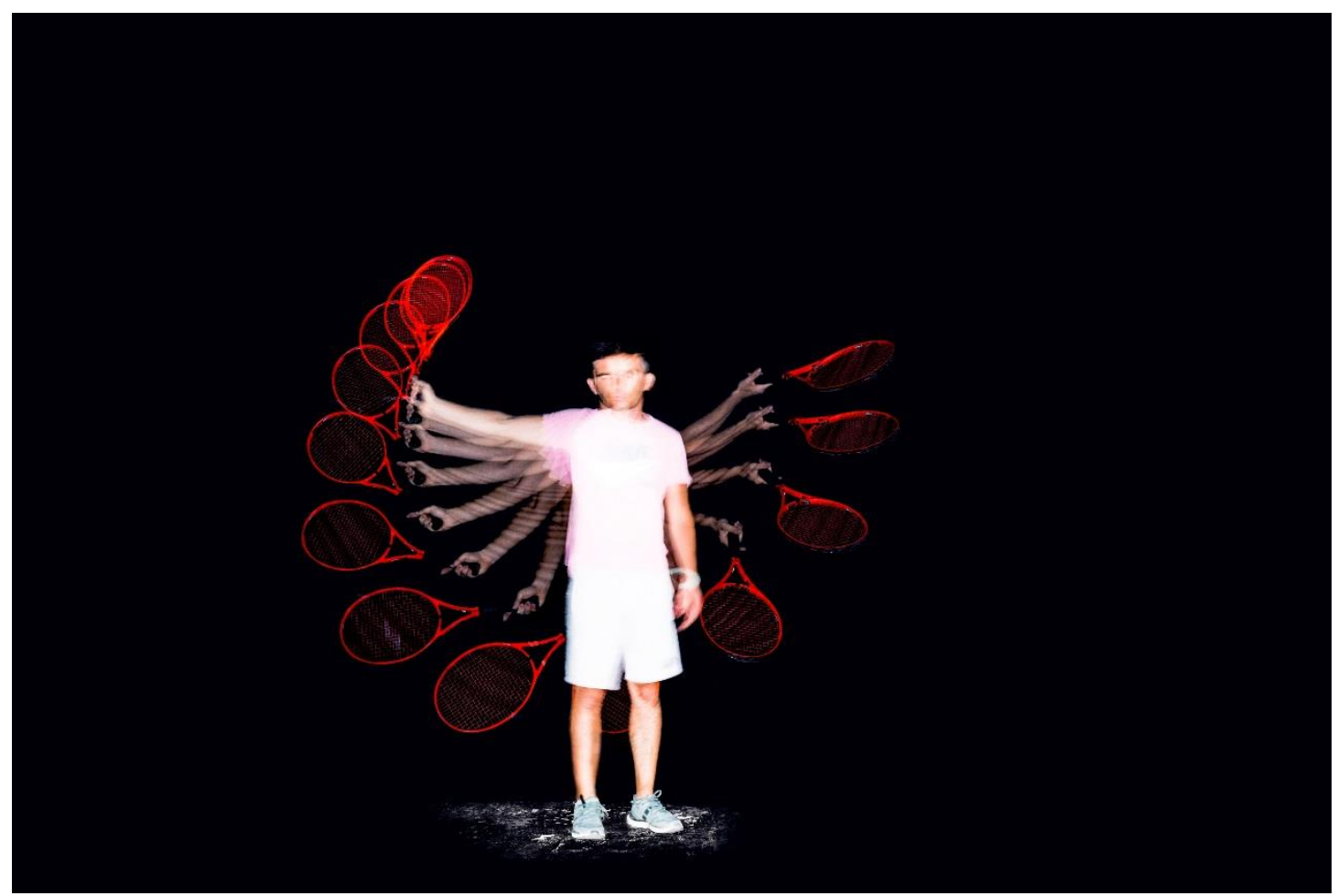

Fig. 8. The forehand underspins' Operating Principle in the frontal plane (rotation: counter clockwise)

In the sport of tennis there is a lack of properly structured biomechanical models. In popular magazines, what is presented as "technique" (in the most general sense) is just a video analysis of some player, exactly the way mathematical methodology forbids us to do. I mean, it is OK for a popular magazine to present a case study, but in no way can observations or measurements, accompanied by numbers and equations, be called "models" and have any regulatory power from then on. This is a logical fallacy known as "begging the question" and its formal formulation is:

$$
\forall((A \rightarrow B) \wedge B) \rightarrow A
$$

Which is a foul interpretation of the valid modus ponens:

$$
\vdash((A \rightarrow B) \wedge A) \rightarrow B
$$

In the literature then, not much can be found regarding tennis biomechanics leading to some kind of proposed technical form, save from observations, measurements and some references to biomechanical principles in general (Elliott et al., 1989; Kleinöder, 1997; Bahamonde, Knudson, 1998; Elliott et al., 2003; Martin et al., 2013; Grambow et al., 2020).

Taking as a starting point Classic Epistemology, a new Paradigm is unveiling in these pages, the first step towards a complete biomechanical model for all tennis strokes. Measurements cannot help in the development of this model; only afterwards technicians are free to measure any player's movement which they think is a good interpretation of this model. Statistics and approximation theory are valid mathematical fields but have nothing to do with either the development or with the evaluation of a model. Similarly, without the prior existence of models, what kind of error do we measure? We set to trace deviation from what? This self-defeating, circular rationale led Classic Greeks to develop the axiomatic method and epistemen to, actually, bypass such problems. The problem was not and is not the accuracy of the measurements. Merely using complicated mathematical formulas to describe a phenomenon is called quasi-mathematicity and is an epistemological fallacy - certainly not a way to increase the validity of a model (Papageorgiou, Lekkas, 2020). The problem lies in the idea of going from measurements and phenomena back to

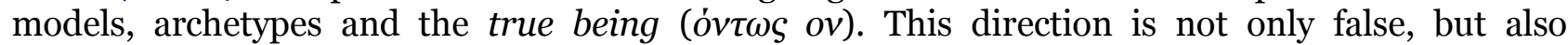
witchcraft and it sets science's clock back more than 2500 years.

Or have we gotten over the Classic Greek Epistemology by relapsing to even more ancient worldviews? If this is the case, why do science in the first place and not go even further back in time 
and perform shamanic rituals instead? As I have repeatedly supported, shamanic rituals seem to be more environmentally friendly than many modern scientific practices.

The reason I insist so much in methodology is that a question asked correctly directly points to the correct answer. It is crucial to understand why we ask questions this way and why epistemology works like that: if correctly structured models are rare in sports science, epistemological discussions are even more rare - even absent.

As far as some additional practical implications are concerned, one should keep in mind the general workflow: players use their most basic understanding (through sensing) and perform the shot mechanisms in the form of some simple exercises. Then, we establish a common understanding through specific anchors, called the reference points of tennis shots (not described here). With the sensorial understanding provided by the shot mechanism and the clearer mental representation of the important parts of the shot's technical model provided by the reference points, we can now communicate effectively in order to introduce the next tool, i.e. the shotteaching mechanism which is how we will develop a meaningful technical form. From then on, "knowing" or "understanding" the form of one or more tennis shots, it doesn't mean we have learned the game of tennis. To achieve that, we introduce drill-synthesis, which, for example, includes random and blocked practice. Drill-synthesis helps players to combine shots or to move while hitting the ball. However, hitting shots while moving is not the game of tennis, in the same way that effectively pressing different keys on a keyboard to write correct words does not make someone a writer or a novelist. So, the next step is drill-structure which goes far beyond the scope of this paper. I just attempted to describe a simplified whole-picture of the process - a process that includes even more tools in the complete tennis Distal Method (e.g. motowords, two double models for motor skills and physical form development, two periodisation tools called performance spirals).

\section{Impact and medical perspectives}

This paper, points towards a holistic training system which is based on a sound biomechanical model produced in the way theory dictates, that is, not based on observations but based on initial conditions (Papageorgiou, Lekkas, 2018). For tennis, it is the first time a model of this kind has been proposed.

Optimal biomechanics is necessary for both performance and injury prevention (Joshi et al., 2011; Kancherla et al., 2014; Ae, 2020). Any training system should have at least two fundamental components: a sound biomechanical model and a method for teaching that model; the former belongs to the domain of biomechanics and the latter to the domain of motor learning and performance. The Distal Method has these two components in its very core, supported by other important tools (related to physical form, periodisation, pedagogy, mental skills training etc.).

\section{Conclusion}

The general concept of "technique" is broken down into biomechanics, bridging/operating principles and technical form and the relationships among these, oftentimes confused concepts have been attempted to be cleared. In this paper, our focus was on the bridging principles. These same principles may also be presented using much heavier mathematical jargon, a task fit for anyone interested; however, my hunch is that such an endeavour would not shed more light, but rather formalise more what has been described here in a simpler geometrical manner, which is also mathematical. Future research should concern the development of models for technical form within the paradigm presented in these few pages. An interesting question that may be answered in another paper would be if there is also a bridge between the final product described in this paper (technical form) and the technical style (a concept which has not been mentioned at all). Technical style is the personalised type of technical form that results after years of training of the technical form and after the individual has adapted the form to their own body-levers, preferences etc. One could say that a specific technical form is characteristic of a certain School, whereas a specific technical style is characteristic of specific individuals (usually expert players). Technical form is taught directly whereas the technical style develops "naturally". So, can we conceive of a bridging principle between these two concepts? 


\section{References}

Ae, $2020-A e, M$. (2020). The next steps for expanding and developing sport biomechanics: Winner of the 2019 ISBS Geoffrey Dyson Award. Sports Biomechanics. Routledge. DOI: 10.1080/14763141.2020.1743745

Bahamonde, Knudson, 1998 - Bahamonde, R., Knudson, D. (1998). Kinematic analysis of the open and square stance tennis forehand. Medicine \& Science in Sports \& Exercise. 30(Supplement): 29. DOI: 10.1097/00005768-199805001-00165

Bloomfield, Ackland, 1994 - Bloomfield, J., Ackland, T.R. (1994). Applied Anatomy \& Biomechanics in Sport. Blackwell.

Elliott et al., 2003 - Elliott, B. et al. (2003). Technique effects on upper limb loading in the tennis serve. Journal of science and medicine in sport/Sports Medicine Australia. 6(1): 76-87.

Elliott et al., 1989 - Elliott, B., Marsh, A.P., Overheu, P. (1989). A Biomechanical Comparison of the Multisegment and Single Unit Topspin Forehand Drives in Tennis. Journal of Applied Biomechanics. 5(3): 350-364.

Grambow et al., 2020 - Grambow, R., O’Shannessy, C., Born, P., Meffert D., Vogt, T.(2020). Serve efficiency development at wimbledon between 2002 and 2015: A longitudinal approach to impact tomorrow's tennis practice. Human Movement. 21(1): 65-72. DOI: 10.5114/hm.2020.88155

Joshi et al., 2011 - Joshi, M., Thigpen, C.A., Bunn, K., Karas, S.G., Padua, D.A. (2011). Shoulder external rotation fatigue and scapular muscle activation and kinematics in overhead athletes. Journal of athletic training. 46(4): 349-57.

Kancherla et al., 2014 - Kancherla, V.K., Caggiano, N.M., Matullo, K.S. (2014). Elbow injuries in the throwing athlete. The Orthopedic clinics of North America. 45(4): 571-85. DOI: 10.1016/j.ocl.2014.06.012

Kleinöder, 1997 - Kleinöder, H. (1997). Quantitative Analysen von Schlagtechniken im Tennis: intra- und interindividuelle Studien bei Spielern unterschiedlichen Leistungsniveaus. Köln.

Knudson, 2007 - Knudson, D.V. (2007). Fundamentals of biomechanics. 2nd edn. Springer.

Martin et al., 2013 - Martin, C. et al. (2013). Upper limb joint kinetic analysis during tennis serve: Assessment of competitive level on efficiency and injury risks. Scandinavian journal of medicine \& science in sports. DOI: 10.1111/sms.12043

Papageorgiou, 2016 - Papageorgiou, K.G. (2016). An Analysis of the Kinetic Chain Model In Forehand Drive. Medicine \& Science in Tennis. 21(2): 22-26.

Papageorgiou, 2019 - Papageorgiou, K.G. (2019). The Distal Method: from psychomotor education to motor expertise. Journal of Physical Education and Sport. 19(1): 633-644. DOI: 10.7752/jpes.2019.01092

Papageorgiou, 2020a - Papageorgiou, K.G. (2020). Exploiting the cognitive-physicalemotional interconnection: motowords and the Distal Method. Journal of Applied Sports Sciences. 1: 113-124. DOI: 10.37393/JASS.2020.01.9

Papageorgiou, 2020b - Papageorgiou, K.G. (2020). On the methodology of Sports Biomechanics. Epistēmēs Metron Logos. (5).

Papageorgiou, 2020c - Papageorgiou, K.G. (2020). Online coach development and certification programs in sports: are they possible? Central European Journal of Sport Sciences and Medicine. 32(4).

Papageorgiou, 2020d - Papageorgiou, K.G. (2020). Online Coach Development Components: the Tennis Distal Method Coach Development. Pedagogy. 92(7s): 17-28.

Papageorgiou, 2020e - Papageorgiou, K.G. (2020). The Distal Method Coach Development for tennis: a new Paradigm in Coach Education. Sport Science. 13(2): 89-101.

Papageorgiou, Lekkas, 2020 - Papageorgiou, K.G., Lekkas, D. (2020). Verification in theory and in the sciences. Epistèmēs Metron Logos. (3): 25-48. DOI: https://doi.org/10.12681/eml.22106

Papageorgiou, Kapadopoulos, 2018 - Papageorgiou, K.G., Papadopoulos, S. (2018). Theatre pedagogy and the Distal Method in psycho-social development (Greek). Applied Pedagogy (Gr.), (9): 1-19.

Papageorgiou, Lekkas, 2018 - Papageorgiou, K., Lekkas, D.E. (2018). On the Methodology of the Analytic Method: Historical Account, Epistemological Suggestions, Stages. Epistēmēs Metron Logos. (1): 70. DOI: 10.12681/eml.19244 